\title{
Study of the Dielectric Behaviour of Pure Polypropylene (PP) and Polypropylene Banana Fiber (PP-B) Composites
}

\author{
Md. Nazrul Islam ${ }^{1}$, M. A. Gafur ${ }^{2, ~ *, ~ A m i r ~ H o s s a i n ~ K h a n ~}{ }^{3}$ \\ ${ }^{1}$ Depertment of Physics, Primeasia University, Banani-Dhaka, Bangladesh \\ ${ }^{2}$ Pilot Plant \& Process Development Center, Bangladesh Council of Scientific and Industrial Research, Dhaka, Bangladesh \\ ${ }^{3}$ Department of Physics, Jahangirnagar University, Savar, Bangladesh
}

Email address:

n_islam58@yahoo.com (M. A. Gafur)

${ }^{*}$ Corresponding author

To cite this article:

Md. Nazrul Islam, M. A. Gafur, Amir Hossain Khan. Study of the Dielectric Behaviour of Pure Polypropylene (PP) and Polypropylene Banana Fiber (PP-B) Composites. Engineering Physics. Vol. 4, No. 1, 2020, pp. 1-6. doi: 10.11648/j.ep.20200401.11

Received: February 21, 2020; Accepted: March 6, 2020; Published: May 28, 2020

\begin{abstract}
This paper reports the dielectric properties of banana fiber reinforced thermoplastic composites. In this research synthetic polymer, polypropylene was used matrix and natural fiber, banana fiber was used as the reinforcing material. Polypropylene $\left[-\mathrm{CH}_{2}-\mathrm{CH}_{2}-\mathrm{CH}_{2}\right.$.]n and different fiber content (wt.\%) of polypropylene-banana fibers (natural fiber) composites were fabricated using a hot-press moulding system. The short fiber percentages was varied as $0 \%, 10 \%, 20 \%$, and $25 \%$ with the matrix materials. The optimum fabrication parameters were established (initial pressure, temperature, etc.), and also optimum short fiber percentage was selected. These composite test samples were fabricated so the short fibers were randomly oriented in the matrix. The electrical properties like dielectric constant and dielectric loss tanjent were measured by a dielectric measurement set up. The measurement was performed over a wide range of frequency of $60 \mathrm{~Hz}$ to $3 \mathrm{MHz}$ and temperature range from $303 \mathrm{~K}$ to $383 \mathrm{~K}$. The results obtained for the composite varied with the variation of fiber percentage. Experimental results of the dielectic properties of pure polypropylene and polypropylene-natural fiber composites were compared. It has been established that the fabricated composition changes its insulating property after adding the natural fibers. The certain percentage of natural fiber addition gives the better dielectric properties and dialectic tanjent.
\end{abstract}

Keywords: Ac Conductivity, Dielectric Constant, Hopping Conduction, Interfacial Polarization, Composites

\section{Introduction}

Natural fibers are the most common reinforcing fibers for polymer matrix composites due to their low cost, high electrical resistance, highest specific strength and densities compared with inorganic fibers and are used in many highvolume applications [1]. A number of natural fibers are available in Bangladesh. They can be used as reinforcing fibers due to their biodegrading and environment friendly characteristics [2]. These polymer-natural fiber composites can be used as electrical device and dielectric materials. These products have greater prospect in their use in material applications where relativity low density of the natural fibers is a major advantage. The present study has thus undertaken to measure the various electrical characteristics (ac conductivity, dielectric, loss tangent) of pure polymer and polymer-fiber composites and investigate the suitability of these composites to use as better dielectric material.

The electrical conduction in polyethylene, a polymer having major use in cable industry, has been investigated as a functional of applied electric field and thickness by Sing and Singh [2]. Where the conduction was found to be ohmic in low field $(400 \mathrm{KV} / \mathrm{m})$ and space charge limited in intermediate fields $(400-1600 \mathrm{KV} / \mathrm{m})$. Newspaper fiberreinforced polypropylene composites were made by applying newspaper sheet directly to polypropylene [3]. Experimental result revealed that the composites made from this method were twice as strong as the polypropylene without newspaper. Abdullah and Das - Gupta [4] studied the electrical, dielectric and pyroelectric properties of three types of ceramic polymer composites. It was found that the dielectric loss of the ceramic- polymer composites was observed to be dominated those of the polymer, while the ceramic phase may have a 
significant conduction on the steady state electrical conduction and low frequency dielectric loss at high temperature. Gahleiner et al. [5] presented a comparative study of electrical and mechanical properties of PP with talk as mineral filler. The determination of liner visco-elastic properties provides a quick and reliable way to investigate filler properties, dispersion and quality. Electrical properties of coir-fiber reinforced low density polyethylene composites were measured by Augstime and Sab [6]. It has been found that the dielectric constant increases with the increase of fiber mixing and decreased with the increase of frequency for all composites. The volume resistively decreases with the increase of fiber content. Characteristics of natural fiber reinforced polyurethane composites were analyzed by Arauji and Reis [7]. Composites with coir fiber or beggase of sugarcane were prepared by melt mixing method. The result shows thermal resistance of both composites of different properties was similar with onset around 573K. Electrical properties of polymer blend with LDPE and Nylon - 7 were measured by Shahin. [8]. It was found that ac conductivity for LDPE was lower than Nylon-6. The electrical properties i.e. volume resistivity dielectric permitivity and dielectric loss factor as well as thermally stimulated depolarization current were measured on PP - Polycarbonate (PC) blends by Myslinski et al. [9]. The results confirmed the existence of some interactions between the non-compatible components of PP-PC blends. It was observed that the volume resistivity varies proportionally to the blend composition. Dielectric loss factor has sharply increases above $403 \mathrm{Kas}$ for pure PC and should reach the maximum at glass transition temperature $\mathrm{T}_{\mathrm{g}}$ of PC. The polymers usually behave as insulating materials having resistivity of the order of $10^{8}-10^{14}$ $\Omega-m$. They have high degree of toughness flexibility and can be moulded at higher temperature. Due to this reason some polymers have high resistivity, high dielectric breakdown strength. In this article we report the electric and dielectric Properties of pure PP and PP-J composites.

\section{Experimental}

\subsection{Materials}

PP was collected from local market. Banana fibers were collected locally. All these fibers were chopped and kept at $383 \mathrm{~K}$ for 24 hours for moisture free. For getting uniform length, all these fibers were sieved by $2 \mathrm{~mm}$ sieve. PP matrix materials were also taken as moisture free at the same procedure.

\subsection{Sample Preparation}

\subsubsection{Fiber Cutting and Mixing}

All the fibers were collected locally and chopped by sharp knife and sieve by $2 \mathrm{~mm}$ sieve to get uniform fiber. This fiber kept at a dry environment (oven) for 24 hours at $383 \mathrm{~K}$. Fiber and polymer were thoroughly mixed using a blender. The mixing time and blade speeds were 2 minutes and $400 \mathrm{rpm}$ respectively. For uniform mixing this process was repeated 3 times and the intermingled fibers are separated manually.

\subsubsection{Casting, Moulding and Demoulding}

A special device is made by steel to very close tolerance for the moulding process. The mixture of fiber and matrix is cast by simply pouring the mixture into the mould and leveling it to the desired thickness. Only slight stamping or hammering on the mould is required for sufficient compaction. The mixed fiber and polymer were taken in a mould. An initial of $50 \mathrm{KN}$ pressure over the sample area was given to top of the mould and then it was kept in a $\mathrm{P} / \mathrm{O} /$ Weber Pressen Hydraulic Machine. The applied pressure is measured by using a load cell, set in the device. Heating was done electrically and the temperature set at $453 \mathrm{~K}$. Only 25-30 minutes were required to reach such temperature. The temperature was kept at $453 \mathrm{~K}$ for 20 minutes. After completion of heating the final pressure of $50 \mathrm{KN}$ over the sample area was set to avoid the kind of voids and have a desired thickness. This additional pressure was kept for one hour. An attempt has been made to prepare PP- fiber composite of different proportion of $\mathrm{PP}$ and fiber according to $(100-X)$ PP: $X$ Fiber $[X=0,10,20$, and 25]. Four batches of different mixing ratios (100:0), (90:10), (80:20), (75:25) were used. The percentage (\%) of fibers was taken as 0,10 , 20 , and 25 of total $100 \mathrm{gm}$ of composites for the fabrication of PP and banana fiber composites. Cooling was done by water flow through the outer area of the heating plates of the hydraulic press machine for 25 minutes.

When the Weber Pressen hydraulic machine is made the specimen, the specimen was cooling, the specimen adheres very strongly with the mould; the makeshift device did required removing by a set up device, which is made as a makeshift device. After cooling and de-moulding the prepared samples were then made in a circular shape to form compact pellets of diameter $8 \mathrm{~mm}$ to. $01 \mathrm{~m}$ by cork-borer with heavy pressure.

\subsection{Testing Procedure}

For ohomic contact both sides of the prepared samples were polished by sand paper and coated with silver paste. The ac. conductivity and dielectric properties have been measured using a Wayne Keer B224 Universal (transformer ratio arm) bridge. The ac conductivity, dielectric constant, dielectric loss tangent are calculated from the measured data. These parameters have been measured at temperatures of 303, 343, $363 \mathrm{~K}$ as a function of frequencies of $60 \mathrm{~Hz} 10 \mathrm{KHz}, 1 \mathrm{MHz}$ and $3 \mathrm{MHz}$. The sample temperature, during the experiment PID thermostatic Oven (SE-70, electrode AS 20438) containing the sample holder which is connected with proper screening to the measuring electrodes. in instruction manual. All this measurements were taken 4 times for each batch and average values were considered. In this measurement there were no major variations with in individual data.

Here the value of conductance $\mathrm{G}_{0}$ was obtained initially by adjusting the conductance ratio arms and after connecting the sample a final value of conductance $G /$ was obtained by adjusting the conductance ration arms. Finally the 
conductance $G_{x}$ was obtained conductance ration $G_{r}$ and $(G$ $\mathrm{G}_{0}$ ). Simultaneously the value of capacitance $C_{x}$ and $G_{x}$ value the calculation for dielectric constant, loss tangent and ac conductivity were performed at different frequencies and temperatures by using the following formulae:

Dielectric constant $\varepsilon=(\mathrm{d} / \mathrm{A}) \mathrm{C}_{\mathrm{x}} / \varepsilon_{0}$

Dielectric loss tangent, $\tan \delta=\mathrm{G}_{\mathrm{x}} / 2 \pi \mathrm{fC}_{\mathrm{x}}$

Where, $\mathrm{d}$ is the thickness, $\mathrm{A}$ is the area of sample and $\varepsilon_{0}$ is permittivity permittivity of free space.

\section{Results and Discussion}

\subsection{Conductivity}

Log ac conductivity vs. inverse temperature at different fiber percentages are shown in figure 1. It is observed that ac conductivity increases with frequency and fiber content (wt. \%) of the composites and also with the working temperatures for all the samples relation may describe such dependence is given by $\sigma \alpha \omega^{\mathrm{n}}$. The values of the exponent $n$ were derived from the slopes of logarithmic conductivity verses different frequencies. The describe values of $n$ were less than unity (shown in table 1) for all temperature ranges may be attributed to the decreases of distribution of relaxation times dominated by interfacial type of mechanism. Similar result was observed by Arauji and Reis [7]. It is also observed that ac conductivity decreases with the increase temperature up to $363 \mathrm{~K}$ and then remains almost constant in the composites at all frequencies. the conductivity becomes less dependent on frequency in going from lower to higher temperature. It is interesting to note that the conductivity is more temperature dependent. Thus it becomes apparent Electrical conduction in these composites is probably due to both ions and electrons. This conductivity dependence on frequency is an indication of hopping conduction. A similar frequency dependence of the ac conductivity in $\mathrm{Zn}-\mathrm{PC}$ has been observed by Saleh et al. [12].

The activation energies can be obtained from the slope of the linear portion of $\log \sigma_{\mathrm{ac}}$ against $(1 / \mathrm{T}) \times 10^{4}$ plots for low and high frequency region as shown in figure 1 . It is observed that the enhancement of activation energy in going from low to high frequency may arise due to the ion - jump mechanism. The activation energies for all composites at low and high frequencies are given in table 2. This may be due to restriction of ion jump for higher concentration of fiber in PP. The differences in activation energies become less with the increase of concentration of fiber in the matrix.

Table 1. $n$ values for all samples.

\begin{tabular}{llll}
\hline $\begin{array}{l}\text { Description of } \\
\text { the materials }\end{array}$ & $\begin{array}{l}\text { Temperature } \\
\text { in }{ }^{\circ} \mathbf{C}\end{array}$ & $\begin{array}{l}\text { The value of } \\
\text { n at } \mathbf{3 3 0 ~} \mathbf{~ H z}\end{array}$ & $\begin{array}{l}\text { The value of } \mathbf{n} \\
\text { at } \mathbf{3 ~} \mathbf{~ M z z}\end{array}$ \\
\hline & 30 & 1.25 & 0.99 \\
PP & 70 & 0.88 & 0.96 \\
& 90 & 0.66 & 0.85 \\
& 110 & 0.54 & 0.80 \\
$10 \%$ PP-B & 30 & 0.98 & 0.99 \\
& 70 & 0.77 & 0.89 \\
& 90 & 0.64 & 0.75 \\
& 110 & 0.44 & 0.52 \\
$20 \%$ PP-B & 30 & 0.99 & 0.85 \\
& 70 & 0.80 & 0.72 \\
& 90 & 0.75 & 0.61 \\
\hline
\end{tabular}

Table 2. Activations energies at low and high frequency regions for PP, PP-J composites.

\begin{tabular}{lll}
\hline $\begin{array}{l}\text { Description of the } \\
\text { materials }\end{array}$ & Frequency region & $\begin{array}{l}\text { Activation energy } \\
\text { Kcal/Mole }\end{array}$ \\
\hline \multirow{2}{*}{ PP } & Low & 4.93 \\
& High & 5.32 \\
$10 \%$ PP-B & Low & 7.714 \\
& High & 10.56 \\
& & 2.56 \\
$20 \%$ PP-B & Low & 5.43 \\
& High & 6.39 \\
$25 \%$ PP-B & Low & 6.63 \\
& High & 6.78 \\
\hline
\end{tabular}



Figure 1. Log $6 a c(\mathrm{ohm}-\mathrm{m})^{-1}$ vs inverse of temperature $\left(K^{-1}\right)$ graph for PP and PP-J composites at low and high frequency.

\subsection{Dielectric Constant}

The plots of dielectric constant against log frequency at different constant frequencies and temperatures are shown in figures $2,3,4,5,6$, and 7 . It is seen that the dielectric constant decreases with increasing frequency and temperature in all the samples. The dielectric constant of all the composites is strongly depends on temperature and frequency. The dielectric constant decreases up to $363 \mathrm{~K}$ and then saturates. The higher 
temperature behavior may be attributed to the thermal agitation, which does not allow polarization in the composites. With higher fiber concentration. Similar result was observed by Krasaz [13]. The dielectric constant values are low and tend to be the same for all samples at higher frequency because at higher frequency the interfacial polarization is less sensitive for rapidly changing electric field. This leads to decrease in the dielectric constant. The dielectric constant is independent of frequency and temperature at about $1 \mathrm{MHz}$. This may be understood from the pinning of dielectric. Similar results were also observed PP-talc-composites by Mamun et al. [14]. The dielectric constant also increases with the concentration of fibers.

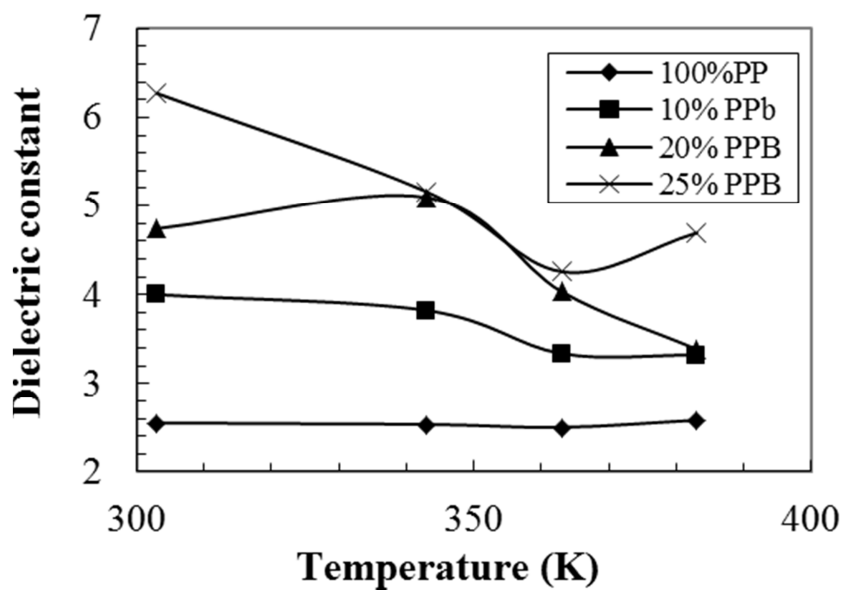

Figure 2. Effect of temperature on dielectric constant at $60 \mathrm{~Hz}$.

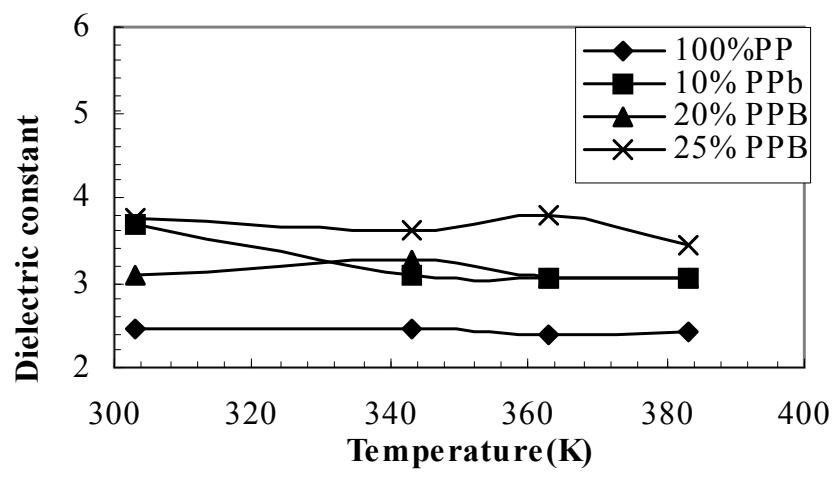

Figure 3. Effect of temperature on dielectric constant at $60 \mathrm{~Hz}$.

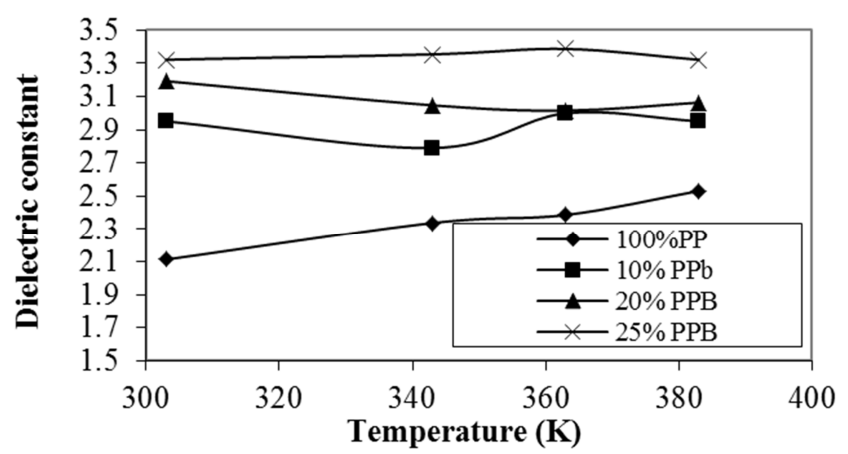

Figure 4. Effect of temperature on dielectric constant at $1 \mathrm{MHz}$.

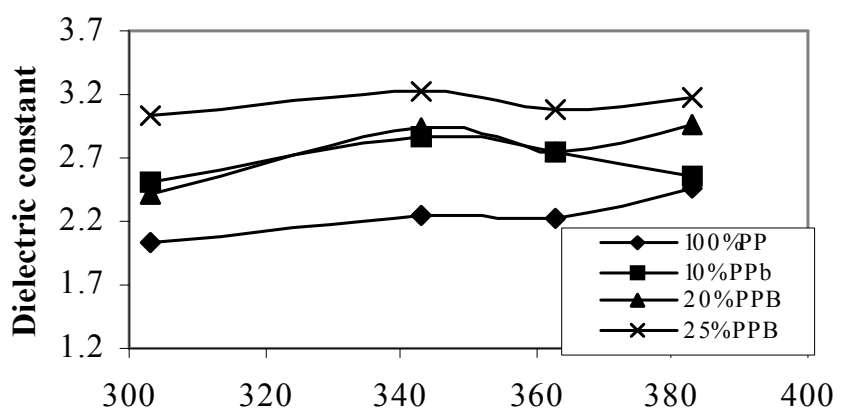

Temperature $(\mathrm{K})$

Figure 5. Effect of temperature on dielectric constant at $3 \mathrm{MHz}$.

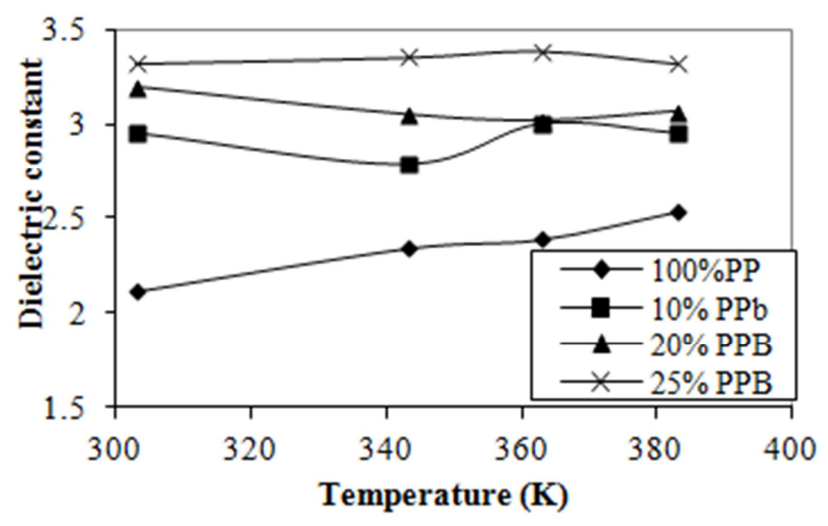

Figure 6. Effect of temperature on dielectric constant at $1 \mathrm{MHz}$.

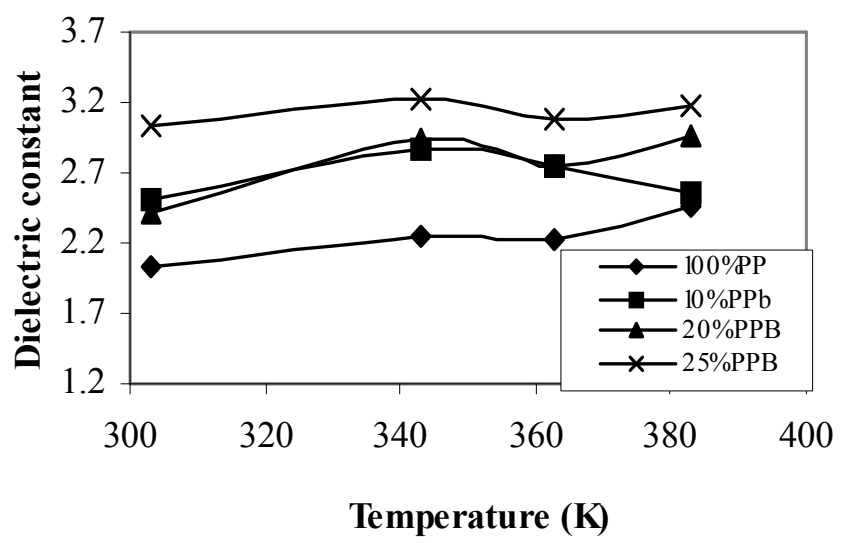

Figure 7. Effect of temperature on dielectric constant at $3 \mathrm{MHz}$.

\subsection{Dielectric Loss Tangent}

The plots of dielectric loss tangent $(\tan \delta)$ vs frequency at different temperatures are plotted in figures 8, 9, and 10, respectively. It is observed that $\tan \delta$ increases with the increasing amount of fiber. With the increase of temperature the dielectric loss tangent decreases for each composite. It is also seen that $\tan \delta$ becomes more dependent at low frequencies with the additional fiber in PP. This is an indication of increase of interfacial type of relaxation in the composites with the increase of fiber. This may be due to diffusion of polymeric chains at the interface of PP and fiber. To calculate the activation enthalpy for all the samples, the logarithm of the frequency corresponding to maximum $\tan \delta$ against the reciprocal of the absolute temperature 
is plotted in figure 4. A straight line was obtained for each composite. From the slope of the straight line the activation enthalpy $(\nabla \mathrm{H})$ is obtained from the relation.

$$
\mathrm{f}_{\mathrm{m}}=\mathrm{A} \exp [-\nabla \mathrm{H} / \mathrm{RT}]
$$

Where $f_{m}$ is the frequency of the maximum loss, $R$ is gas constant, $\mathrm{T}$ is the absolute temperature. The activation ent values involved in the dielectric relaxation process of the pure polypropylene and composites materials are in table 3 .

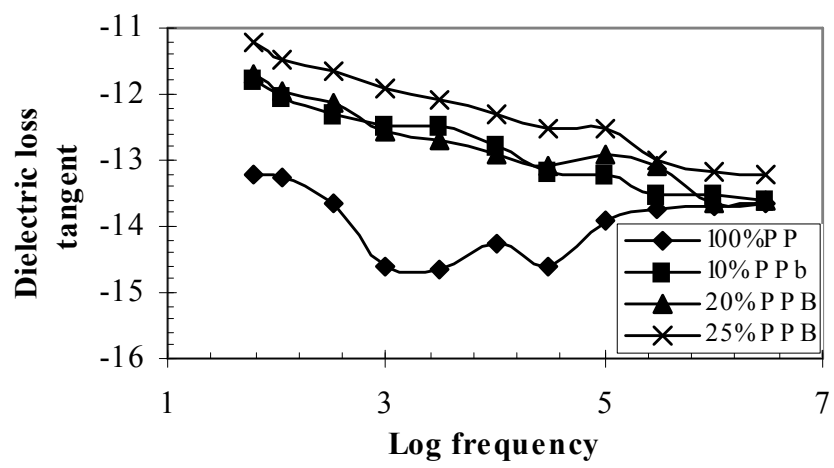

Figure 8. Effect of frequency on dielectric loss tangent loss tangent of PP-B Composites at $303 \mathrm{~K}$.

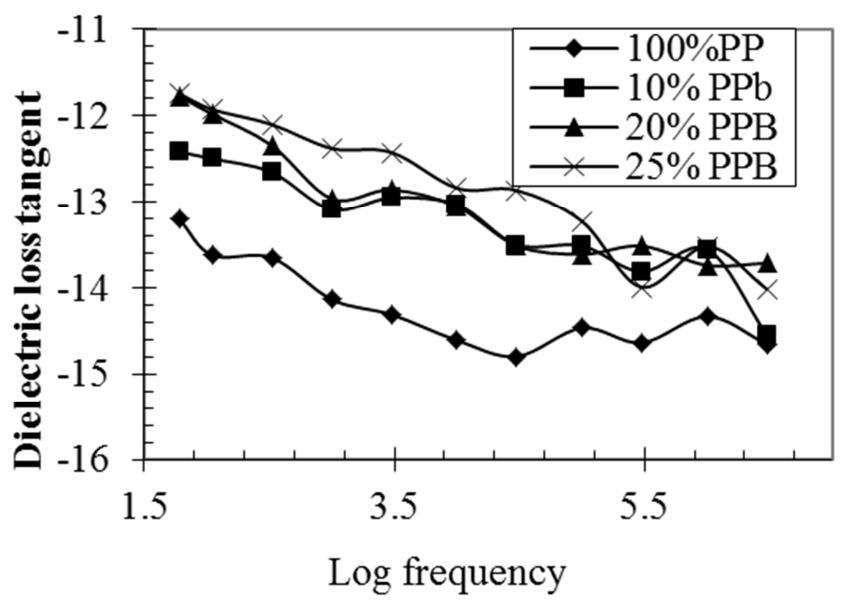

Figure 9. Effect of frequency on dielectric loss tangent loss tangent of $P P-B$ Composites at $343 \mathrm{~K}$.

Table 3. Activation enthalpy for PP and PP-J composites.

\begin{tabular}{ll}
\hline Description of the materials & Activation enthalpy (Kcal/mole). \\
\hline PP & 25.489 \\
$10 \%$ PP-B & 20.36 \\
$20 \%$ PP-B & 13.091 \\
$25 \%$ PP-B & 17.775 \\
\hline
\end{tabular}

It is observed that the activation enthalpy goes on decreases with increasing amount of fiber in the composites. The decrease in activation energy may be due to change in internal viscosity of the samples at higher percentage of the fiber where chains are separated. On increasing the amount of fiber the free volume increases and hence rotation of dipole is more easy. This may reduce the activation energy as described by Moh and Devis [15].

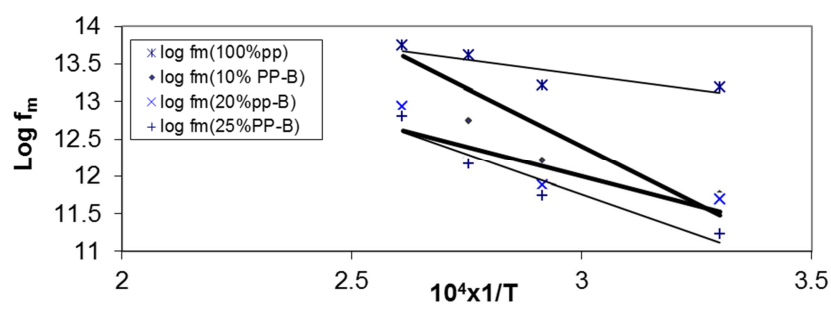

Figure 10. $\log f_{m} V_{S}$ inverse of tempt. graph for $P P \& P P-B$ composites at $303 K$.

\section{Conclusion}

The frequency and temperature dependence of $\sigma_{\mathrm{ac}}$ may be attributed to the increases of relaxation times dominated by interfacial type of mechanism. The dielectric constant decreases with frequency and temperature. This behavior may be attributed to the thermal agitation, which does not allow polarization. Also the dielectric constant increases with the increasing of fiber, which indicates that fiber act as an intermediate plasticizer in the composites. The variation of $\tan \delta$ with frequency and temperature is used and the nature of the composition dependence is similar to that of the ac conductivity. Experimental observations suggest that it is possible to make PP-banana fiber composites by the hot-press moulding method and these composites can be used in the manufacture of high frequency electrical device. This fabrication technique may also be applied to prepare the dielectric material for the preparation of capacitor and other electrical devices.

\section{References}

[1] M. M. Mohanty, and G. Hinrichsen, Macromol. Matter. Eng. 276/277, (2000) 1-24.

[2] S. Sing and P. Singh, Ind. J. Pure and Appl. Phy., 28 (1990) 490.

[3] Shan Ren and N. S. Hons Devid, J. Renforced Plastics and Composites: 12, (1993) 1311-1322.

[4] M. JAbdullah and D. K. Das-Gupta, IEEE Transaction on Electrical Insulations, 25, (1990), 605.

[5] Gahleiner, M. K. Bernrlitner and W. Neible, J. Appl. Polym. Sci 53. (1994) 284-289.

[6] Paul Augstime and Thomas Sab, J. Dept. Chem. Bharatama Coll, Kerala 68201, India. (1994).

[7] De Arauji and Carla Reis, UFRJ-DE Technologia 21949-900, Brasil.

[8] N. Shahin, Investigation of the ac electrical properties of LDPE and Nylon -6 blends, M. sc thesis University of Dhaka, Bangladesh) (1993).

[9] P. Myslinski, Z. Dobkowaki and B. Krajehski, Polymer Blend processing, Morphology and Preparations. 2Eds, E. Martus Celli, R. Palumbo and M. Krysze wski, (London: Plenum press ny) pp. 157-163 (1984).

[10] T. Umemura, T. Suzuki and T. Kashi wazaki, IEEE Transalation on Electrical Insulation, Vol. E1-17. No. 4 August (1986). 
[11] C. R. Ashcraft and R. H. Boyd, J. Polymer Sci. Polm. Phys. Ed. 14, (1976) 2153.

[12] A. M. Saleh, RD Gould and A. K Hossan, Phy Sat Sol (A) 139 (1993) 334.

[13] F. E Krasaz, Dielectric Properties of Polymers (New York: pleanum press) p 17 (1972).
[14] Al-Mamun, Hosne Jahan Rahman and A. K. Roy, A Study of the ac electrical properties of pure propypolylene and propypolylene-talc composites (Dhaka University Journal.) Ssci. 51 (1) 163-168 (2003 January).

[15] N. F. Moh and E. A. Devis, Electronic Process in Non Crystalline Materials (Oxford: Clarendom - Press) 1979. 